\title{
Relationship between the Working-day and Day-off Physical Activity of Adults in Hong Kong
}

\author{
Wong S.L. , Lee L.Y.K., Lam S. C., Yeung C. Y. \\ Division of Nursing and Health Studies, The Open University of Hong Kong, Hong Kong, China
}

Copyright (C) 2015 by authors, all rights reserved. Authors agree that this article remains permanently open access under the terms of the Creative Commons Attribution License 4.0 International License

\begin{abstract}
This study aims to assess the relationship of the levels of physical activities among working adults over a period of seven consecutive days, between working days and days off. This cross-sectional study used a sealed pedometer to measure the physical activity of working adults in terms of their number of daily walking steps on working days and days off. A voluntary sample of 745 working adults aged 18 - 64, who were recruited from different occupational categories in Hong Kong, had completed the study. The participants were fairly active when compared against the recommended levels of physical activity. The average daily walking steps reached 8,400 over a period of seven consecutive days. A significant difference was found between the number of walking steps on working days and that on days off. Two groups of working adults who walked less at work were plant and machine operators and assemblers, and clerks. A significant positive correlation was also found between physical activities on working days and those on days off. One's walking steps on working days were found to significantly increase one's walking steps on days off when the effect of age and gender were controlled in the analysis of multiple regressions. Physical activities on a working day are likely to determine those on a day off. Sedentary work habits may induce sedentary activities on days off. The results inform us that health promotion campaigns, for allowing exceptional levels of activities at workplaces should be undertaken to encourage working adults in Hong Kong.
\end{abstract}

Keywords Physical Activity, Walking, Working Adult, Hong Kong

\section{Introduction}

Hong Kong has been being transformed from a manufacturing to a service economy since the 1990s [1]. Most jobs in Hong Kong involve providing assistance, such as in finance and accounting, legal consulting for business, and tourist accommodation, rather than in manufacturing.
Thus, the working adults in Hong Kong have been becoming more sedentary on working days because of the changing nature of their jobs. About $52 \%$ of the population is a part of the labour force; these people spend more than one third of their daily life at work. Thus, the participation of working adults in physical activities is notably declining [2-3].

The Behavioural Risk Factor Survey of 2012 revealed that Hong Kong residents are inactive because 50.1\% do not engage in vigorous physical activities for five days or more per week [4]. De Cocker, Cardon, and De Bourdeaudhuij [5] examined pedometer-determined physical activity levels in 1,239 Belgian adults. This sample recorded an average of 9,655 steps daily, and $58.4 \%$ of the participants were partially active as compared to the pedometer index of 10,000 steps daily. De Cocker et al. found that daily step levels significantly varied with employment status (i.e., self-employed versus employed by others). The performance of physical activity is considerably controlled not only by personal intention but also by social context (e.g., occupational circumstances).

Occupations have manifest and latent features that determine the physical activities of the worker, but cannot be modified. However, no study has reported on the relationship between occupations and daily physical activities as defined by pedometers among working adults. Investigating the relationship between physical activities during working days and that during days off among Hong Kong working adults from nine occupational categories could determine the nature of occupations that influences the physical activities of working adults.

\section{Physical Activity}

Physical activity is defined as any bodily movement produced by skeletal muscles and resulting in energy expenditure. Physical activities can be categorized into three main types, namely, occupation, leisure, and sleep [2]. Optimal energy expenditure is related to weight control and balance as well as vital organ endurance. The amount of physical activity during both working and leisure time is the 
key determinant of energy expenditure. A healthy lifestyle recommendation should be that of at least 30 minutes of moderate-intensity type activity on most, and preferably all days of the week [4]. This practice can improve cardiorespiratory endurance, muscular endurance and strength, and body composition and flexibility. Regular physical activities effectively reduce the risk of obesity and prevent chronic illness, such as cardiovascular disease, osteoporosis, and diabetes mellitus [6].

In past epidemiological studies [2-3], the participation in physical activity was measured by self-reporting. This method involved a questionnaire that asked about the time retrospectively spent in physical activities during free time, at home, at work, and during travel in the last seven day period. Therefore, associations of being physically inactive with bad health outcomes may be due in part to recall bias in reporting the physical activities performed in the last seven days. To avoid similar interpretation problems, we used an alternative approach to address the question of how the physical activity could be measured prospectively and objectively.

The most common form of physical activity among adults is walking. Therefore, the level of physical activity may be expressed in terms of the number of walking steps per day [7]. Given that pedometers accurately assess step-counts as a daily physical activity pattern, Tudor-Locke and Meyers [8] proposed pedometer indices of physical activity levels that could be used in research. Working adults with 10,000 walking steps per day can be considered regularly active; those with 6,000 to 9,999 walking steps per day as fairly (somewhat) active; and those with 5,999 or fewer walking steps as inactive. Tudor-Locke and Bassett [9] found a correlation between daily walking step-counts and health, and recommended walking 10,000 steps daily to maintain health. This pedometer index has been adopted worldwide as a recommended level of healthy physical activity.

Occupation significantly affects the physical activity of a working adult. Daily physical activities significantly vary with job characteristics. McCormack, Giles-Cortic, and Milligan [10] examined adults who were walking 10,000 steps daily in a statewide cross-sectional physical activity survey. These adults used a pedometer for one week and recording their daily activities in a journal. The performance of the physical activity in terms of walking steps was measured. McCormack et al. concluded that the workplace physical activity and blue-collar occupations facilitated the daily achievement of 10,000 steps among male workers. However, increased sedentary work tasks foster one to be inactive and so not reaching the daily achievement of 10,000 steps. The effects of highly sedentary work have been a concern that might be seen as causing the problem of obesity. Physical inactivity leads to being overweight and obesity, which are the fifth greatest risks for life-threatening diseases, such as cardiovascular disease [11].

\section{Conceptual Framework}

The social ecological approach is an essential framework that considers the social contextual determinants such as several occupational circumstances which may be causing disparities in health [12-13]. The levels of physical activity of working adults may vary considerably from person to person and with a given set of working conditions. Kirkcaldy and Furnham [14], and more recently Guest [15], suggested two models for examining an individual's work behavior that influences their day-off activity levels: generalization and segmentation. The generalization model holds that one's work practices can influence one's life experiences (i.e. one's free time) in either a positive or a negative way. Kirkcaldy and Furnham illustrated how there is a positive correlation between occupational and off-work activities so that both are reduced. In contrast, they suggested that antisocial people gravitate toward off-work activities in such a way as to have a negative correlation with their occupation [14-15]. The segmentation model explains that practices at work and during free time are two distinct domains of life that are independent and unrelated [14-15].

Many groups of working adults are presented with diverse behavioral options in their workplaces. However, the difference in the physical activity level among different occupational categories and between working days and days off remains unknown. The relationship between occupational and off-work physical activity has not been compared. Thus, a systematic approach is warranted in studying the relationship between the physical activity of working adults on working days and that on days off in Hong Kong. If a working adult constrained by work conditions becomes fairly physically active on working days, will the adult transfer their work habit to their days off? We hypothesized that the physical activity (i.e., number of walking steps) of working adults on working days was correlated positively with those on days off.

\section{Aims and Objectives}

This study aimed to assess the relationship of physical activities of working adults in a period of seven consecutive days, including working days and days off. The objectives of the study were as follows: (1) to assess the overall walking steps of working adults over seven consecutive days; (2) to examine the difference in the number of walking steps of working adults in nine occupations in Hong Kong on working days and days off; (3) to assess the relationship between physical activity during working days and during days off among the working adults of nine occupations.

\section{Methods}

\subsection{Design}

The study was a cross-sectional survey. Data were collected through a self-reporting questionnaire and a pedometer, from a volunteer sample from varying occupations. The questionnaire was used to collect the 
demographic information about the participants. The daily number of walking steps was measured and recorded as representing the daily level of physical activity. Each participant was instructed to wear a pedometer, which directly recorded the physical activity of the working adults over seven consecutive days.

\subsection{Participants}

By sending invitation letters to Trade Unions registered in Hong Kong [16], the members who were employees were approached via their Trade Unions. Participation in the research was completely voluntary that is, the participants could refuse to participate or withdraw any time without consequences or loss of benefits. For a significance test of a sample, the product-moment correlation coefficient $r$ is a small effect (0.1), alpha is at 0.05 , and the necessary sample size for the power of 0.8 was 783 [17]. The participants were employed in any of the nine occupational categories in Hong Kong according to the Labour and Social Welfare Bureau of Hong Kong [18]. These categories were: (1) managers and administrators, (2) professionals, (3) associate professionals, (4) clerks, (5) service and shop sales workers, (6) craft and related workers, (7) elementary occupations, (8) plant and machine operators and assemblers, and (9) skilled agricultural and fishery workers. The data collection process was approved by the committee for research and development of the associated university.

\subsubsection{Inclusion Criteria}

Participants should fulfill the following criteria: including being aged 18 to 64; could walk independently; being employed full-time in any of the above occupational categories, and would have taken days off as usual during the period of seven consecutive days.

\subsubsection{Exclusion Criteria}

Participants had been working for seven consecutive days over the study period.

\subsection{Measurement}

The distinction between physically active and inactive participants was based on the participant's self-reporting in the questionnaire and the sealed pedometer record of walking steps.

\subsubsection{Self-reported Questionnaire}

The questionnaire included questions relating to demographic information. Variables of interest to this study were those such as age, gender, educational level and occupation. Occupational category was an independent variable considered to influence physical activity levels. Participants within the same occupational category may have comparable levels of physical activity in similar work settings.

\subsubsection{The Sealed Pedometer}

A sealed pedometer was used to assess the number of daily walking steps as being the level of physical activity. This aimed to avoid participants being motivated to not walk as usual. The sealed display of the step-count on the pedometer minimized the bias toward participants who would increase their daily walking steps during the study period. The Omron (HJ-113) pedometer is statistically valid and accurately counting the steps tallied with the controlled distance assessed during a treadmill and over ground walking [6, 19]. The pedometer is valid, reliable, and practical and does not affect walking behavior; thus, it can be used to assess the level of physical activity under free living conditions [6].

\subsection{Data Collection Procedures}

The purpose of the study was explained to the participants by five research nurses. The participants were asked about their demographic information in the questionnaire. The participants agreed to have their personal information used for the purposes of this research. The participants were instructed to wear the pedometer, either on the right or left hip, for seven consecutive days, except while bathing, swimming, cycling, or sleeping. The pedometer step-count display was sealed with opaque adhesive tape; therefore, the step-count was to be kept as being unknown by the participant during the seven consecutive day period. Throughout the period, the participants daily recorded whether the day was a working day or a day off. The day after the assigned period, research nurses visited the participants to retrieve the records of their walking steps from the sealed pedometer and the daily log.

\subsection{Statistical Analysis}

The Statistical Package of Social Sciences (SPSS, Version 19) was used to analyze data. Descriptive statistics, including frequencies, percentages, means and standard deviations, and a coefficient of variation (CV), were used to summarize the characteristics of the participants and their walking steps over the seven consecutive day period. Inferential statistics were also used for analysis. A paired t-test was performed to verify the difference in mean scores of walking steps on a working-day and a day off for each occupational category. A Pearson correlation was used to test the relationship of the walking steps between working days and days off for each occupational category. A hierarchical multiple regression was used to test variables (i.e. walking steps on working days and occupational categories) to be able to explain the variance of walking steps on days off, when the effect of age and gender were controlled for. The significance level was set to $<0.05$. 


\section{Results}

The study was based on the 919 recruited participants, of which 807 completed the questionnaires and complied with the instructions on wearing the pedometer (completion rate $=$ 88\%). However, 62 participants did not take a day off because they were required to work overtime during the period. These participants were excluded from the data analysis. No significant difference was found in the walking steps on working days between the excluded and included participants. The data collected from the 745 included participants were then analyzed.

Table 1 shows the distribution of pedometer results according to the demographic data from all the participants. The overall physical activity in terms of the walking steps of working adults for seven consecutive days was 8,400 steps. The 55-64 age group recorded the highest, with 10,000 steps on average and with the highest variance ( $S D=3.7)$, whereas the other age groups had practically the same number of walking steps of 8,100 to 8,900 steps on average. The daily number of walking steps for males was higher than that for females.

Significant differences were found between the walking steps on working days and those on days off among all participants $(t=14.23, p=0.001)$ in Table 2. A Coefficient of Variation (CV) could determine the distribution of variables for walking steps on working days and days off among the working adults In general, the walking-steps on working days with the smaller CV (30.7\%) is less dispersed than the walking-step on days off with a larger CV (50.1\%). Five types of employees who walked less at work were identified: (1) plant and machine operators and assemblers, (2) clerks, (3) managers and administrators, (4) professionals, and (5) associate professionals. These employees walked 7,600 to 8,600 steps. For craft and related workers, plant and machine operators and assemblers, and skilled agricultural and fishery workers, no significant difference in the average number of steps was found between working days and days off. On their days off, craft and related workers walked 8,200 steps which was the highest on average compared with other occupations and the participants as a whole.

Table 1. The distribution of pedometer results according to the demographic data of all participants ( $\mathrm{N}=745)$

\begin{tabular}{|c|c|c|c|c|}
\hline \multirow[t]{2}{*}{ Variables } & \multirow[b]{2}{*}{$\mathrm{N}$} & \multirow[b]{2}{*}{ (\%) } & \multicolumn{2}{|c|}{ Walking Steps Per Day* } \\
\hline & & & Mean & (SD) \\
\hline \multicolumn{5}{|l|}{ Age group (Years) } \\
\hline $18-24$ & 48 & $(6.4)$ & 8.4 & (2.6) \\
\hline $25-34$ & 350 & $(47)$ & 8.3 & $(2.2)$ \\
\hline $35-44$ & 183 & $(24.6)$ & 8.1 & (2.3) \\
\hline $45-54$ & 120 & $(16.1)$ & 8.9 & (2.8) \\
\hline 55-64 & 42 & $(5.6)$ & 10.0 & (3.7) \\
\hline Missing & 2 & $(0.3)$ & & \\
\hline \multicolumn{5}{|l|}{ Gender } \\
\hline Female & 374 & $(50.2)$ & 8.1 & $(2.2)$ \\
\hline Male & 371 & $(49.8)$ & 8.8 & (2.8) \\
\hline \multicolumn{5}{|l|}{ Educational Level } \\
\hline Primary or below & 68 & $(9.1)$ & 8.3 & $(2.7)$ \\
\hline Secondary & 202 & $(27.1)$ & 8.7 & (2.9) \\
\hline Tertiary or above & 475 & (63.8) & 8.4 & (2.3) \\
\hline Total & 745 & $(100)$ & 8.4 & (2.5) \\
\hline
\end{tabular}

*Results in thousands of steps per day

Table 2. Descriptive statistics, mean differences and correlation of walking steps per day for the working-day and day-off among the occupations

\begin{tabular}{|c|c|c|c|c|c|c|c|c|c|}
\hline \multirow[b]{3}{*}{ Variables } & \multirow[b]{3}{*}{$\mathrm{N}$} & \multicolumn{4}{|c|}{ Walking Steps Per Day* on } & & & & \\
\hline & & \multicolumn{2}{|c|}{ Working-day } & \multicolumn{2}{|c|}{ Day-off } & \multicolumn{2}{|c|}{ Paired $t$} & \multicolumn{2}{|c|}{ Pearson } \\
\hline & & $\mathrm{M}(\mathrm{SD})$ & CV\% & $\mathrm{M}(\mathrm{SD})$ & $\mathrm{CV} \%$ & $t$ & $\begin{array}{c}p- \\
\text { value }\end{array}$ & $r$ & $\begin{array}{c}p- \\
\text { value }\end{array}$ \\
\hline Managers and administrators & 40 & $8.3(2.2)$ & 26.5 & $7.1(2.6)$ & 36.6 & 2.53 & 0.01 & 0.14 & $>0.05$ \\
\hline Professionals & 120 & $8.5(2.5)$ & 29.4 & $6.9(3.2)$ & 46.4 & 5.34 & 0.001 & 0.37 & 0.001 \\
\hline Associate professionals & 197 & $8.6(2.5)$ & 29.1 & $6.4(3.5)$ & 54.7 & 8.61 & 0.001 & 0.29 & 0.001 \\
\hline Clerks & 122 & $8.1(2.3)$ & 28.4 & $6.6(3.2)$ & 48.5 & 5.28 & 0.001 & 0.34 & 0.001 \\
\hline Service workers and shop sales workers & 152 & $9.6(3.0)$ & 31.3 & $7.5(3.7)$ & 49.3 & 7.20 & 0.001 & 0.44 & 0.001 \\
\hline Craft and related workers & 47 & $9.3(3.2)$ & 34.4 & $8.2(4.3)$ & 52.4 & 1.86 & $>0.05$ & 0.43 & 0.003 \\
\hline Plant and machine operators and assemblers & 16 & $7.6(2.6)$ & 34.2 & $7.0(3.6)$ & 51.4 & 0.67 & $>0.05$ & 0.39 & $>0.05$ \\
\hline Elementary occupations & 48 & $9.1(3.1)$ & 34.1 & $6.9(4.2)$ & 60.9 & 4.06 & 0.001 & 0.52 & 0.001 \\
\hline Skilled agricultural and fishery workers & 3 & $8.9(0.5)$ & 5.6 & $4.0(2.8)$ & 70.0 & 3.27 & $>0.05$ & 0.51 & $>0.05$ \\
\hline Total & 745 & $8.8(2.7)$ & 30.7 & $6.9(3.5)$ & 50.1 & 14.23 & 0.001 & 0.38 & 0.001 \\
\hline
\end{tabular}

*Results in thousands of steps per day 
Table 3. Reporting a multiple regression model with explanatory variables of walking steps on the day-off

\begin{tabular}{|c|c|c|c|c|c|c|c|c|c|c|}
\hline \multirow[b]{2}{*}{ Variables } & \multicolumn{5}{|c|}{ Model 1} & \multicolumn{5}{|c|}{ Model 2} \\
\hline & $\begin{array}{l}\text { Coefficient } \\
\text { (ßeta) }\end{array}$ & $\begin{array}{l}\text { Standard } \\
\text { Error }\end{array}$ & $95 \%$ CI & $\begin{array}{l}\text { Wald } \\
\left(X^{2}\right)\end{array}$ & $\begin{array}{c}P \\
\text { value }\end{array}$ & $\begin{array}{c}\text { Coefficient } \\
\text { (ßeta) }\end{array}$ & $\begin{array}{l}\text { Standard } \\
\text { Error }\end{array}$ & $95 \%$ CI & $\begin{array}{c}\text { Wald } \\
X^{2}\end{array}$ & $\begin{array}{c}P \\
\text { value }\end{array}$ \\
\hline Constant & 4962.2 & 500.0 & $3980.6-5943.8$ & 9.9 & $<0.001$ & 1751.9 & 1054.5 & $-318.4-3822.2$ & 1.7 & 0.09 \\
\hline Age & 46.2 & 13.3 & $20.05-72.26$ & 3.5 & 0.001 & 30.2 & 13.2 & $4.2-56.2$ & 2.3 & 0.02 \\
\hline Gender $^{a}$ & 611.4 & 258.0 & $104.9-1117.8$ & 2.4 & 0.018 & 233.3 & 253.6 & $-264.6-731.1$ & 0.9 & 0.34 \\
\hline Walking steps on working days & & & & & & 0.5 & 0.04 & $0.4-0.6$ & 10.5 & $<0.001$ \\
\hline \multicolumn{11}{|l|}{ Occupations $^{\mathrm{b}}$} \\
\hline Managers and administrators & & & & & & -35.9 & 968.8 & $-1937.9-1866.1$ & -0.04 & 0.97 \\
\hline Professionals & & & & & & -131.2 & 880.8 & $-1860.4-1598.0$ & -0.2 & 0.88 \\
\hline Associate professionals & & & & & & -622.9 & 872.3 & $-2335.5-1089.7$ & -0.7 & 0.48 \\
\hline Clerks & & & & & & -235.8 & 892.5 & $-1988.1-1516.4$ & -0.3 & 0.79 \\
\hline Service workers and shop sales workers & & & & & & -84.6 & 878.6 & $-1809.4-1640.3$ & -0.1 & 0.92 \\
\hline Craft and related workers & & & & & & 526.0 & 945.8 & $-1330.7-2382.8$ & 0.6 & 0.58 \\
\hline Elementary occupations & & & & & & -733.4 & 946.8 & $-2592.2-1125.4$ & -0.8 & 0.44 \\
\hline Skilled agricultural and fishery workers & & & & & & -3658.3 & 2045.6 & $-7674.3-357.7$ & -1.8 & 0.07 \\
\hline
\end{tabular}

Note: $\mathrm{R}^{2}=0.03$ for Model $1, \Delta \mathrm{R}^{2}=0.17$ for Model $2(\mathrm{p}<0.001)$

b The occupation of plant and machine operators was set as baseline group against which all other occupation should be compared

A significant positive correlation ( $r=0.38, p=0.001$ ) was also found between the walking steps of the adults on working days and days off. A positive correlation was consistent and significant in the various occupational categories, except for managers and administrators, plant and machine operators and assemblers, and skilled agricultural and fishery workers. A hierarchical multiple regression was used to assess the ability of the two independent variables (the walking steps on working days and the nine occupational categories) to explain the dependent variable of walking steps on days off, after controlling for the influence of age and gender. Preliminary analyses were conducted to confirm there was no violation of the assumptions of normality, linearity, multicollinearity and homoscedasticity. Age and gender were entered in Model 1 (see Table 3), thereby explaining 3\% of the variance in the walking steps on days off. After the entry of the walking steps on working days and eight dummy variables for occupational categories in Model 2, the total variance explained by Model 2 as a whole was $17 \%, F(9,731)=14.1, p<0.001$. In Model 2, only the two independent variables were statistically significant, with the walking steps on working days recording a higher beta value (beta $=0.5, \mathrm{p}<0.001$ ) than for age (beta $=30.2, \mathrm{p}<0.02$ ) in Table 3 . It also indicates that as the variable for the walking steps on a working day (beta $=0.5$ ) increases by one step in Model 2: walking steps on days off could increase by 0.5 steps which was statistically significant. After we controlled the effect for age in Model 2, age $(b=30.2)$ could explain a significant amount of variance in walking steps on days off. It indicates that as the age increased by one year, there were an extra 30.2 walking steps on days off. Finally, no significant occupational category contributed to explain the variance of walking steps on days off. 


\section{Discussion}

In this study, we assessed the overall physical activity of working adults in Hong Kong in terms of their number of walking steps over seven consecutive days. The working adults are seen as 'fairly active' against on recommended physical activity levels [8-9] because not all participants achieved the recommended 10,000 steps per day. The overall physical activity level of the participants was similar to those observed in developed countries, specifically among 4,000 participants who were fairly active according to the same recommendation [20-21]. The relationships between the social-demographic variables and walking behavior are similar to those described in other studies [20-21]. Walking behavior is related to age and gender; that is, older adults are more likely to walk as much as possible daily, than younger ones. Males prefer to have more physical activities, such as walking, than females.

This study firstly evaluated the overall physical activity in terms of walking steps on both the working days and days off of adults from different occupational categories. Working adults in Hong Kong walk less frequently on their days off than on their working days. Plant and machine operators and assemblers, as well as clerks, in Hong Kong walk minimally at work. Craft and related workers walk maximally on days off. Walking steps on working days were less distributed compared with those on days off among the occupations; therefore, the working-day physical activity has become to be seen as more functional and unmodifiable. Similar relationships were consistently found in various occupational categories, except among the managers and administrators, plant and machine operators and assemblers, and skilled agricultural and fishery workers.

The hypothesis of the study was evaluated. The physical activity (i.e., number of walking steps) of working adults on working days is related positively with those on days off, which is supported by the model of generalization as mentioned [14-15]. The positive correlation between walking steps on working days and those on days off indicates that working adults are likely to transfer their habit of physical activity at work to their days off. This finding implies that the physical activities of these workers on working days could influence their physical activities on days off, even when variables such as their ages and genders were both controlled. It was not surprising that the work demand from different occupational categories could not explain the influences on day-off habits among adults. The demand of many jobs, such as customer services, has been as a result of an intensifying in the speed of responses to an information load rather than in the physical strength to manufacture any product in recent decades [1]. The habit of physical activity on working days, which can be seen to be decreasing among various occupations, is likely to be transferred to those on days off.

However, a few limitations must be considered. The participant recruitment strategies, exclusion of participants, and limitations of the pedometer may have influenced the recorded number of walking steps. First, the participants volunteered to participate in the study - the idea of volunteers suggesting that they might have higher levels of physical activity than the average person even though the participant recruitment efforts were uniform across the nine occupational categories. The number of participants was significantly different from among the nine occupational categories. Associate professionals formed the largest group of participants (26\%). Only $0.1 \%$ of the participants were skilled agricultural and fishery workers. We aimed to recruit a similar number of participants from all the nine occupational categories. However, the number of skilled agricultural and fishery workers has been decreasing since the 1980s. In future studies, collaboration with various occupational unions could enhance the representation of participant recruitment.

Second, the exclusion of participants without days off may have removed the participants offering most valuable information on how their occupations have affected their physical activity. Thus, we may have underestimated physical activity on working days. Further research should also follow up on the overtime issue arising from within this study.

Third, the pedometer is limited to only measuring walking steps to reflect participants' physical activity levels. Participants may be lacking in opportunities for walking; in terms of demand or satisfaction; participants might be joining in with other activities after work. However, the participants were instructed to take off their pedometers during such activities as cycling and swimming. We suggest that participants should be asked to have a logbook recording their activities in any future study. Furthermore, several participants who followed such instructions forgot to wear the pedometer again after the excluded activities. We suggest that a positioning sensor with alerting signals be integrated into the pedometer. This sensor could indicate whether the pedometer had been taken off. In future studies, the user-friendly modification of pedometer parameters could facilitate the accurate measurement of physical activity.

\section{Conclusions}

In general, working adults are fairly physically active. The major finding of this study is that decreasing physical activity levels on a working day are likely to reproduce the same level on days off. Predominantly sedentary work may translate into similar sedentary activity levels on days off. Our finding has important implications for employees and organizations. If a job comprises sedentary works an individual finds undesirable in regards to health, an individual has to be able to make an informed employment decision. On the other hand, individuals may accept a job characteristic that cannot be changed if faced with such barriers as sitting for long hours in the workplace [13]. Organizations need to consider practices that could help 
ameliorate associated physical inactivity in workplaces. The results inform us that health promotion campaigns on exceptional activity levels should be conducted for working adults with increased sedentary work tasks in Hong Kong. Organizational policies on allowing flexible times for regular exercise in workplaces can facilitate and motivate working adults to engage in more physical activities on working days and possibly increase such activities on days off.

\section{Acknowledgements}

The work described in this paper was supported by the Open University of Hong Kong Research Grant (No.2009/1.1).

\section{REFERENCES}

[1] Information Services Department, Hong Kong Special Administrative Region, (2012). Hong Kong as a Service Economy. Retrieved from

http://www.gov.hk/en/about/abouthk/factsheets/docs/service _economy.pdf

[2] Leisure and Cultural Services Department, HKSAR, (2009). Sport for All - Participation Patterns of Hong Kong People in Physical Activities consultancy study findings unveiled. Retrieved from

http://www.lcsd.gov.hk/specials/sportforall/pdf/press_release _e.pdf.

[3] Department of Health, HKSAR, (2010). Action plan to promote healthy diet and physical activity participation in Hong Kong. Retrieved from http://www.gov.hk/english/pub_rec/pub_rec_ar/pdf/ncd_ap/ Action\%20Plan_Eng_whole\%20doc.pdf

[4] Centre for Health Protection, HKSAR, (2013). Physical activity. Retrieved from,http://www.chp.gov.hk/en/content/9/25/8804.html

[5] De Cocker, K., Cardon, G., \& De Bourdeaudhuij, I. (2007). Pedometer-determined physical activity and its comparison with the International Physical Activity Questionnaire in a sample of Belgian adults. Research Quarterly for Exercise and Sport, 78 (5), 429-37.

[6] Lam, S. C., Lee, L. Y. K., Wong, S. L., \& Wong, A. K. P. (2012). Pedometer-determined physical activity and body composition in Chinese working adults. Journal of Nursing Scholarship, 44 (3), 205-214.

[7] Jordan, A.N., Jurca, G.M., Tudor-Locke, C., Church, T. S., \& Blair, S. N. (2005). Pedometer indices for weekly physical activity recommendations in postmenopausal women.
Medicine \& Science in Sports \& Exercise, 37 (9), 1627-1632.

[8] Tudor-Locke, C. \& Myers, A. M. (2001). Methodological considerations for researchers and practitioners using pedometers to measure physical (ambulatory) activity. Research Quarterly for Exercise and Sport, 72 (1), 1-12.

[9] Tudor-Locke, C. \& Bassett, D. R. Jr. (2004). How many steps/day are enough? Sports Medicine, 34 (1), 1-8

[10] McCormack, G., Giles-Corti, B. \& Millgan, R. (2006). Demographic and individual correlates of achieving 10,000 steps/day: use of pedometers in a population-based study. Health Promotion Journal of Australia, 17 (1), 43-47.

[11] World Health Organization, (2011). Media centre: Obesity and overweight (Fact sheet N 311). Geneva: World Health Organization. Retrieved from http://www.who.int/mediacentre/factsheets/fs311/en/

[12] Emmons, K. (2000). Health behaviours in a social context. In L. Berkman \& I. Kawachi (Eds.), Social epidemiology (pp. 242-266). New York: Oxford University Press.

[13] Sallis, J.F. \& Owen, N. (2002). Ecological models of health behavior. In K. Glanz, B.K. Rimer \& F.M. Lewis (Eds.), Health behaviour and health education: Theory, research, and practice (3 rd ed.). San Francisco: Jossey-Bass.

[14] Kirkcaldy, B. D. \& Furnham, A. (1990). Personality and sex differences in recreational choices. Sportwissenschaft, 1, 43-55.

[15] Guest, D. E. (2002). Perspectives on the study of work-life balance. Social Science Information 41 (2), 255-279.

[16] Registry of Trade Unions, Labour Department. (2009). 2009 - Annual statistical report of Trade unions and employer's organizations. Retrieved from http://www.labour.gov.hk/tc/public/pdf/rtu/ASR2009.pdf

[17] Cohen, J. (1992). A power primer. Psychological Bulletin, 112, 155-159.

[18] Labour and Social Welfare bureau, HKSAR, (2010). Study on earnings mobility: Executive summary http://www.lwb.gov.hk/eng/other_info/Executive\%20Summ ary\%20_Eng.pdf

[19] Crouter, S.E., Schneider, P. L., Karabulut, M. \& Bassett, D. R. Jr. (2003). Validity of 10 electronic pedometers for measuring steps, distance, and energy cost. Medicine and Science in Sports and Exercise, 35 (8), 1455-1460.

[20] Inoue, S., Ohya, Y., Odagiri, Y., Takamiya, T., Suijo, K., Kamada, M., Shimomitsu, T. (2011). Sociodemographic determinants of pedometer-determined physical activity among Japanese adults. American Journal of Preventive Medicine, 40 (5), 566-571.

[21] Tudor-Locke, C., Bassett, D. R. Jr., Rytherford, W. J., Ainsworth, B.E., Chan, C. B., Croteau, K., ... Wojcik, J. R. (2008). BMI-referenced cut-off points for pedometer-determined steps pers day in adults. Journal of Physical Activity \& Health, 5 (Suppl. 1), 126-139. 